\title{
Single-mode tuning of the plasmon resonance in high- density pillar arrays
}

\author{
Matthew D. Arnold ${ }^{1, *}$ \\ ${ }^{1}$ School of Mathematical and Physical Sciences, University of Technology Sydney, \\ PO Box 123, Broadway, NSW 2007, Australia \\ "matthew.arnold-1@uts.edu.au
}

\begin{abstract}
The Maxwell-Garnett (MG) effective medium model has a pure resonance controlled by volume fraction $f$, but is usually invalid at high density. I present special $2 \mathrm{D}$ structures that match quasistatic MG over the entire range $0<f<1$, in several regular and semi-regular arrays, expanding the applicability of MG. Optimal contours depend on both lattice and fill-factor, transforming from circular at low fo nearly polygonal at high $f$. A key insight is the direct relationship between optimal surface polarization and surface position. Electrodynamic calculations underline the effect of constituent permittivity on spatial dispersion and required sizes for quasistatic response in various materials.
\end{abstract}

\section{Introduction}

Artificially structured materials enable tuning of material properties beyond the limited choices of single constituents, and can be optimized for many functionalities(Torquato et al. 2002). For example, smooth variation in effective refractive index enables optimal flow of light for anti-reflection(Poxson et al. 2009) and cloaking(Pendry et al. 2006). In metal structures, coupling between light and electrons (plasmon waves) offer strong concentration of energy for particle(Miller et al. 2014) and material(Rinnerbauer et al. 2014) absorption. Detailed structural optimization of these systems is computationally expensive so simple models for them are very attractive. The Maxwell-Garnett (MG) effective medium model(Garnett 1904), widely used for over a century, can be intuitively tuned via the average density. However, MG has typically been limited to sparse structures, due to near-field coupling between neighboring parts. In this article I optimize rod contours to MG across all fillfactors for a variety of lattices. The efficiency of optimization is greatly improved by the realization that the optimal contour matches the optimal surface polarization. Later I discuss the limits of validity of the quasistatic MG model, including extensive electrodynamic calculations which confirm that the ratio of period to absolute effective reduced wavelength is strongly correlated with the magnitude of electrodynamic effects.

\section{Maxwell-Garnett model and resonant spectra}

The main property considered in this work is the effective permittivity, which in the quasistatic limit may be defined as

$$
\varepsilon_{e f f}=\frac{\int \varepsilon(r) E(r) d r}{\int E(r) d r},
$$

where $\varepsilon(r)$ and $E(r)$ are the space-varying permittivity and electric field respectively. An effective permeability $\mu_{e f f}$ may be similarly defined, further yielding effective refractive index $n_{e f f}=\sqrt{\mu_{e f f} \varepsilon_{e f f}}$ and impedance $Z_{\text {eff }}=\sqrt{\mu_{\text {eff }} / \varepsilon_{\text {eff }}}$. In non-magnetic bulk materials the permeability is unity in which case $n=1 / Z$, but this is not necessarily true in structured materials. In recent literature the Bloch phase is often included in the homogenization (1), but it is omitted here for several reasons: the phase is explicitly small, the focus is on propagation along the invariant rod axis (no phase delay across the unit cell), and in other directions in lossy composites the definition of periodic phase is complex (these important points will be elaborated later). In the quasistatic limit, Eq. (1) can typically be recast in terms of discrete resonances(Bergman 1979):

$$
\frac{\varepsilon_{e f f}}{\varepsilon_{0}}-1=\sum_{i} \frac{C_{i}}{S_{i}-\left(1-\varepsilon / \varepsilon_{0}\right)^{-1}},
$$

$S$ is the depolarization defining a resonance position, and $C$ is the dipole moment of that resonance. Once mode parameters have been calculated, they can be applied to any number of real materials, even non-conducting constituents (in which case the resonances are virtual). Calculation of the full response of arbitrary structures can be relatively expensive. While several sophisticated models have been developed (e.g.(Sen and Torquato 1989)), 
many parameters are required to account for multiple resonances in Eq. (2). In contrast, the MG model has a single dipole resonance

$$
\begin{gathered}
C_{M G}=f, \\
S_{M G}=L(1-f),
\end{gathered}
$$

but is usually assumed to require a dilute volume fraction $f<<1$ of ellipsoidal inclusions such as cylinders $(L=1 / 2)$. Relating Eqs. (2)-(4), and considering sum rules(Fuchs and Liu 1976), this model is a pure dipole plasmon resonance: $C$ is maximum, and $S$ is maximum for dipoles in isotropic systems.

It is well known that MG is exactly obeyed at inclusion depolarization $L=0$ and $L=1$ (i.e. for laminar geometries and for longitudinal polarization of rods), corresponding to the effective-medium bounds. Instead we will discuss the more interesting cases that occur at intermediate inclusion depolarization, e.g. when electric field is transverse to infinite rods. This case, exemplified in Figure 1, is particularly relevant for construction methods that produce a thin film of vertically aligned rods, with propagation along the rods (normal to the film).

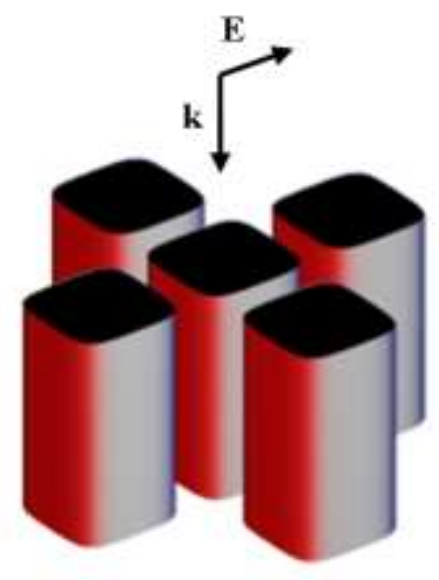

Figure 1 (Color online): Representative simulated geometry: array of infinitely long rods with permittivity $\varepsilon$, with propagation vector $\mathbf{k}$ longitudinal to the rod and electric field $\mathbf{E}$ in the transverse plane.

It is well-known that this type of geometry allows two general situations where MG is inaccurate, neatly summarized in a study of the effect of cylinders (Milton et al. 1981). As fill-factor increases, multimodes are excited and increase in strength and density towards percolation(Perrins et al. 1979; Mcphedran and Mckenzie 1980). At percolation a continuum of modes is formed, and this persists beyond threshold due to cusps formed by the overlap of cylinders (Milton, McPhedran et al. 1981).

The latter case will not be a significant focus in this work, although some results from the literature are of general interest: in particular the alternative Bruggeman model is more appropriate to this situation. This model was fitted to the ellipsometric response of flat (i.e. not pillar) Au square arrays at high fill-factor(De Zuani et al. 2016), although suppression of percolation is mainly due to the flat particles (for the appropriate polarization $L \rightarrow 0$ ) and the fitted fill-factor differs significantly from the geometric fill-factor. The optimization problem for this model has been approximately solved using cuspoid particles in a square lattice(Torquato and Hyun 2001).

In this article the main approach to optimal MG is to suppress multimodes arising from interaction of smooth inclusions. General optimization approaches for extremal properties such as conductivity and/or stiffness have been reviewed elsewhere (Bendsøe and Sigmund 1999; de Kruijf et al. 2007; Cadman et al. 2013), but I will briefly highlight some relevant studies that demonstrate geometries where multimodes are reduced. Isotropic surfaces on cubic lattices (Zhou et al. 2010) nearly span the range between permittivity bounds, and electrical and magnetic properties can also be optimized with more complex geometries (Huang et al. 2012). Multimodes can be reduced in arrays of squares(Markel and Schotland 2012), and permittivity of rounded square(Feng and Whites 2001) and cube(Whites and Feng 2002) arrays is close to MG. The conductivity of punctured sheets is bounded above by MG and is closer for hexagonal than triangular holes(Hetherington and Thorpe 1992; Hyun and Torquato 2000). The spectral reflection edge of metal arrays is controlled by $f$ (Perrins, Mckenzie et al. 1979), and truncated square plasmonic crystals(Rinnerbauer, Shen et al. 2014) have better spectral response than simple squares. None of these observations rigorously demonstrate agreement with the single-mode of the MG model, and many of the optimization studies rely on expensive optimization of voxel-sampled geometries.

However, it has been noted that extremal properties are reached when the polarization field is uniform in the inclusion (Milton 2002). Further, in two-dimensions suitable contours can be systematically determined via 
conformal transformations to periodic slits, which is a Dirichlet problem that can be solved numerically. It was also noted how given solutions can be transformed to different lattices but no demonstrations were shown. Milton highlights that Vigdergauz advanced a different conformal approach, mostly in the context of optimal stiffness in separate lattices, specifically square (Vigdergauz 1994), rhombic(Vigdergauz 1996), and hexagonal/triangular(Vigdergauz 1999). Unfortunately, the methods as presented are non-trivial to implement and it appears that only a few more complex geometries have been attempted on a case-by-case basis. Here we will take an optimization approach, which is relatively straight-forward to apply to semi-regular lattices and is perhaps less expensive than other optimizations, and investigate the significance of electrodynamic effects.

\section{Significance of non-static effects}

In this article, initial exploration is facilitated by the assumption that classical quasistatics is sufficient for optimizing the fundamental dipole response in these arrays. As will be demonstrated, this is accurate when all physical sizes are small compared to the wavelength and skin depth including the effective medium itself. The quasistatic assumption used here is incompatible with perfect conduction assumed in some literature - the applicability and implications of these assumptions will be further discussed in this section. Sizes must also be large compared to quantum smearing lengths - the total effect of electrodynamic and quantum damping can be minimized(Blaber et al. 2009), but high-quality electron conductors are somewhat susceptible. Such calculations are difficult for arbitrary geometries and only classical effects will be considered here.

Electrodynamic effects (such as radiation damping, magneto-electric coupling, non-locality, spatial dispersion, and diffraction) tend to increase with size. However, the onset and significance of these effects fundamentally depends on whether the inclusions are dielectrics or conductors, and the wavelength range (e.g. microwave or visible), as we shall now see. It has long been known that dielectric permittivity affects deviations from MG, which can be predicted through series expansion (McPhedran et al. 1996). More recently it has been realized that more interesting effects become evident at very high permittivity. Dielectric rods exhibit electric and magnetic Mie resonances at reduced wavelengths $\left(\lambda=\lambda_{0} / n\right)$ that scale with the radius(Vynck et al. 2009), with the magnetic resonance occurring at significantly shorter wavelengths. Consequently, in low density rod arrays, negative permeability and negative refraction is observed for in-plane propagation in a narrow band but in this case only at a period that is larger than the reduced wavelength. Similarly, spatial dispersion in seen in large permittivity arrays of spheres(Alù 2011): divergence from MG is readily observed at period:wavelength ratio $>0.02$ if the permittivity is 20 , and at higher permittivity of 120 differences are pronounced around resonances at larger periods.

Perfectly conducting particles are effectively magnetic so in this case it can be shown that permittivity-only MG is insufficient even for sphere arrays of very short periods(Alù 2011). Studies assuming perfectly conducting yet very dilute rods (Belov et al. 2003) assert that spatial dispersion is inevitable and that the effective permittivity along the rods must be modified to account for the wavevector in that direction. However, accumulated literature on wire media was reviewed by several of the same authors (Simovski et al. 2012), where it was noted that for structures smaller than the skin-depth the PEC assumption is inapplicable and spatial dispersion is not apparent. This point is also directly addressed in a computational study(Wells et al. 2014), where it is demonstrated that non-local models based on the PEC assumption fail at optical and near-IR frequencies, and (apart from missing longitudinal modes) local MG is reasonably good at predicting effective propagation constants of transverse EM modes in the long wavelength limit.

Other studies quantify where the quasistatic approximation is applicable for conductors. Calculated propagation in the plane of lossless Drude nearly-touching nanorod arrays show that multipole modes are magnetic, but the fundamental dipole modes closely match electrostatic response for periods as high as $\lambda_{p} / 6$ (Shvets and Urzhumov 2005). It also was stated that sub-wavelength implies $<50 \mathrm{~nm}$ for metals but the origin of this estimate was not elaborated. Plasmonic band structures of Drude inclusions (damping ratio 0.01) showed excellent agreement up to periods of 0.1 wavelengths: including spheres in fcc lattice with $12 \%$ fill-factor (Inglesfield et al. 2004), and cylinders in square lattice with $8 \%$ for transverse polarization and up to percolation for longitudinal (Pitarke et al. 2007). Several studies have considered the accuracy of $\mathrm{MG}$ for $\mathrm{Ag}$ in anodic $\mathrm{Al}_{2} \mathrm{O}_{3}$ with realistic losses. Calculations at $20 \mathrm{~nm}$ period of showed good agreement for fractions up to 30\% (Fang et al. 2009). Oblique reflection measurements showed good agreement for $60 \mathrm{~nm}$ period (and $25 \%$ fill factor) at wavelengths above $600 \mathrm{~nm}$ (Kanungo and Schilling 2010). Calculated normal-incidence refractive index resonant spectra showed reasonable agreement for $60 \mathrm{~nm}$ lattices at 10 and $17 \%$ (Silva et al. 2013).

Considering the evidence in the literature, it is reasonable to concur with the statement that quasistatic behavior should be observed when the period is significantly smaller than the absolute effective wavelength (Elser et al. 2006) - this allows for dielectric wavelength reduction, conductor skin depth, and resonant effects. This requirement further emphasizes the role of damping in determining the minimum effective wavelength, which 
may include contributions from both intraband and interband processes. Considering the transverse MG resonance of rod composites with low-loss Drude inclusions in free-space, it can be shown that the worst-case effective wavelength, which occurs at moderate fill-factors, is $|\lambda|>1.9 \lambda_{p} \sqrt{\gamma / \omega_{p}}$ ( $\omega_{p}$ bulk plasmon, $\gamma$ damping). Since the plasma wavelength of most good conductors is around $100 \mathrm{~nm}$ and damping ratios are of order 0.01 , a minimum effective wavelength in the order of $10 \mathrm{~nm}$ might be expected. However, the contribution of interband absorption in real materials relaxes this constraint somewhat. A survey of actual permittivity tables shows that minimum effective wavelengths for the MG resonances of good conductors (e.g. the coinage metals) are around $40( \pm 10) \mathrm{nm}$, poorer conductors (e.g. the catalyst metals and conducting nitrides) are loosely clustered around $100( \pm 40) \mathrm{nm}$, and for Restrahlen bands (e.g. in SiC) can be on the order of microns. In most metals the minimum effective wavelengths occur at high fill factors - at moderate fill factors such as those shown later, interbands further relax the effective wavelength. In transparent regions the effective wavelength reverts to a fraction of the free-space wavelength.

Production of the shortest implied periods is challenging, but effective medium response near this range have been demonstrated using electrochemical techniques in silicon(Golovan' et al. 2007), alumina(Shinji et al. 1992) and metal(Saito and Miyagi 1989), and oblique deposition for a range of materials and wavelengths(Hodgkinson et al. 2005). Some control over pillar morphology is possible(Hodgkinson, De Silva et al. 2005), suggesting potential for MG-like response at short wavelengths. Current nanofabrication should be feasible for transparent/Restrahlen materials in the near-IR onwards, and recent advances in the area hold some promise for conducting materials.

\section{Method}

Initially a boundary element method (BEM)(Mayergoyz et al. 2005) was used, because it is efficient, can be readily matched to Eq. (2), and allows direct optimization of the contours. Briefly (in 2D),

$$
\begin{gathered}
\sum_{\text {lattice }} \oint \frac{\hat{n} .\left(\vec{r}-\vec{r}^{\prime}\right)}{\left|\vec{r}-\vec{r}^{\prime}\right|^{2}} d r^{\prime} \sigma_{i}\left(\vec{r}^{\prime}\right)=\left(2 S_{i}-1\right) \pi \sigma_{i}(\vec{r}) \\
C_{x y}=\left(\oint r_{x} \sigma d r\right)\left(\oint \sigma^{-1} \hat{n}_{y} d r\right)\left(\oint \sigma^{-1} \sigma d r\right)^{-1} A_{\text {cell }}^{-1}
\end{gathered}
$$

where $\sigma$ is a charge density eigenvector, $\hat{n}$ the outwards facing normal, and $A_{\text {cell }}$ is the area of the unit cell. The equations were discretized with $N$ (typically $3 \times 10^{3}$ per particle) line-segments of length $d r$, and the singularity in Eq. (5) was integrated via closure(Mayergoyz, Fredkin et al. 2005). Eqs. (5) and (6) allow comparison to MG Eqs. (3) \& (4). Subscripts $x y$ denote spatial components: where two are present the first is the detector and the second is the source. In this work most of the lattices are isotropic so $x \& y$ are degenerate.

As detailed in the next section, a suitably parameterized contour was first optimized via a standard simplex algorithm to minimize the deviation of $S$ from the expected MG result. However, the deviation was generally not zero, leaving open the question of whether a perfect match could be achieved. To resolve this question, free optimization on all contours points should be performed, but a naïve optimization is very expensive due to repeated eigenmode calculations for variations in every point on the contour. The efficiency of this final optimization can be greatly increased via an important insight that arises directly from the BEM: the surface charge of a perfect dipole is proportional to the projection of the surface normal to the applied electric field. Alternatively, the target $\sigma_{i}^{-1}$ for (5) \& (6) is the contour position relative to the center of each closed contour, so the contour gradient can be readily established from the left residual $\Delta L$ in the eigenequation (5):

$$
\Delta L=\left(\sum_{\text {lattice }} \oint \frac{\hat{n} \cdot\left(\vec{r}-\vec{r}^{\prime}\right)}{\left|\vec{r}-\vec{r}^{\prime}\right|^{2}} d r^{\prime}+\pi f\right)\left(\vec{r}-\oint_{\text {particle }} \vec{r} d r\right) \rightarrow 0 .
$$

Optimization can then be performed via gradient descent on Eq. (7). Matrix storage can be avoided, since the first order approximation for small isotropic deviations from $\mathrm{MG}$ are:

$$
\Delta C \sim 2 \Delta S \sim-\Delta L . \Delta R /\left(\pi^{2} f^{2} A_{\text {cell }}\right) .
$$

Deviation can be confirmed later by more complete diagonalization. Typically I optimized to less than $10^{-6}$ error. These insights should also be applicable to three dimensions.

In this work I restrict the search to 2D: in this case the lattice sum (5) is related to the conjugate of the Weierstrass zeta function(Abramowitz and Stegun 1964) 


$$
\zeta(z)=\sum_{W=\Omega}(z-W)^{-1}+W^{-1}+z W^{-2},
$$

where $x \& y$ directions are transformed respectively to real and imaginary parts in $z$, summed over the infinite set of complex lattice vectors $\Omega$. Although Eq. (9) is unconditionally convergent, its first term corresponding to the sum in Eq. (5), depends on the boundary shape. Other terms can be removed: $\sum W^{-1} \rightarrow 0$ for large ellipsoidal terminations; $\sum W^{-2} \rightarrow 0$ for isotropic sums and is otherwise easy to calculate. The infinite sum of (5) can then be estimated either by adding $e^{i 2 \vartheta} z \pi / A_{\text {cell }}$ (an elliptical termination aligned with the source direction $\vartheta$ ), or by $\hat{n}_{y} r_{x} d r \pi / A_{\text {cell }}$ (inflation accounting for a finite circular boundary). The 2D sum converges within a few terms of elliptic theta series(Abramowitz and Stegun 1964).

\section{Full-wave electrodynamic methods}

To confirm the validity of the BEM implementation, and investigate the onset of electrodynamic effects, a number of independent simulations on periodic arrays were conducted within the commercial finite-element package COMSOL. One strategy was eigenmode calculations where the effective index was determined from the mode propagation, and the dominant mode identified by electric-field overlap with plane-wave excitation assumed. Another was the plane-wave response of finite slabs (about 10 periods thick and terminated by backgroundmatched layers), including S-parameter retrieval of the effective index(Chen et al. 2004). Although impedance is also available from S-parameter retrieval, in this case it is particularly sensitive to surface termination (especially for conducting and resonant composites), and hence it is not necessarily representative of effective bulk behavior(Simovski 2011). Field homogenization could also be performed and hence the bulk effective index deduced. Calculations were implemented separately for on-axis and in-plane propagation to take advantage of reduced dimensionality.

For propagation along the rods, calculations included TEM mode index of infinite arrays in 2D, as well as calculations on a 3D slab with field homogenization at the mid-plane. Phase was ignored because is common to all fields travelling along the rods. In this direction, for computational efficiency, symmetry was enforced by perfect electric(magnetic) conductor boundaries normal to the electric(magnetic) fields - this does not capture all modes but does allow the important TEM modes, justified due to the symmetry of plane-wave excitation.

For propagation in the plane, periodic boundaries imply that the Bloch phase across the structure should be considered, however its definition in lossy structures requires some discussion. In the photonic crystal literature where lossless materials predominate, it is common to specify the (real) wavevector and determine the (possibly complex) temporal frequency from the eigenmodes. Indeed, this was the approach used in early studies of plasmonic rod media where Drude response was assumed(Smith and Pendry 2006). However, tabulated material properties and optical-frequency plane-wave experiments dictate real temporal frequency so that instead the complex Bloch frequency for a specified direction is calculated giving the effective index directly: special methods are required to implement this within COMSOL(Davanço et al. 2007; Schmidt and Kappeler 2010; Fietz et al. 2011). In this case, field homogenization (particularly for finite slabs) is no longer simple because the phase delay should be included but it is neither fixed nor real. In this direction all calculations can be reduced to 2D.

Modal and finite-thickness COMSOL results agreed and all results converged to the static result as period reduced but, as a further check, selected results were also calculated using well-known codes that employ completely different algorithms. The modes of dielectric inclusions $(\varepsilon=12)$ for all three unique orientations were compared against the plane-wave Bloch-mode code MPB(Johnson and Joannopoulos 2001). The retrieved properties of finite films of Drude inclusions with propagation along the pillars were compared against the Fourier-modal code S4(Liu and Fan 2012).

\section{Results and discussion}

\section{Static results and optimization}

Using the quasistatic method above, contours that achieve MG response can be found: Fig. 1 illustrates that this is primarily achieved by varying the curvature. As expected, circles percolate (Fig. 1a), and polygons generally have strong multimodes (Fig. 1d). A useful starting point for 2D optimization are the Schwarz-Christoffel (SC) contours (Fig. 1b), which conformally fill any polygonal structure(Driscoll 1996). Using SC brings the dipole mode closer to MG, but multimodes can be dramatically reduced by scaling SC for sharper contours (Fig. 1c) and then completely cancelled by a small amount of free optimization. 
(a)

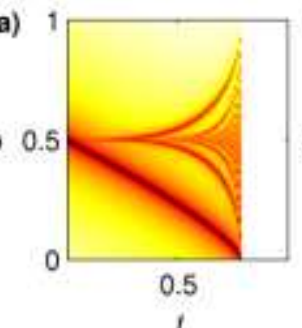

(e)

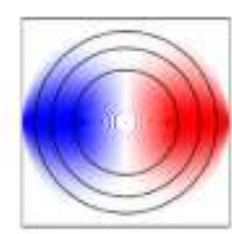

(b)

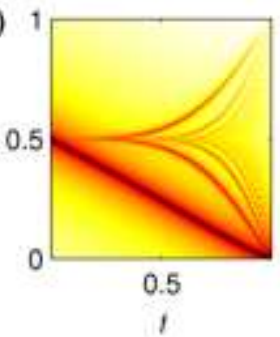

(f)

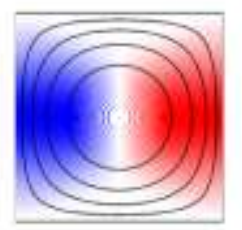

(c)

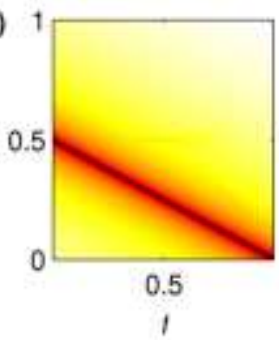

(g)

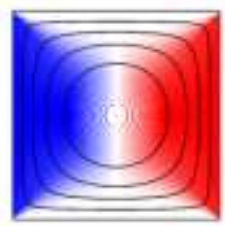

(d)

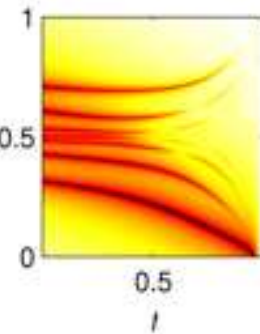

(h)

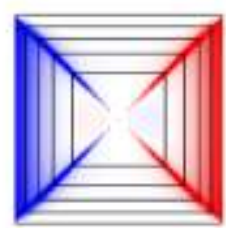

Fig. 2 (color online): Effect of roundness in square lattices, with circular (a,e), conformal (b,f), nearly optimal (c,g), and nearly polygonal $(\mathrm{d}, \mathrm{h})$ contours. Modes are dark lines in (a-d), as a function of fill factor $f$ and depolarization $S$. The dipole is the strongest mode, with MG response in (c). The evolution of dipole surface charge is shown (e-h), with guide contours in black at $20 \%$ intervals in $f$. Circles (a) percolate at $f \approx 78 \%$ in a square lattice.

Concentration of surface charge is strongly correlated with multimode response (Fig. 2), so a balance of electric forces is important for pure dipole response. A circular shape (Fig. 2e) has optimal distribution at low $f$, but near percolation charge concentrates around the gap. Conversely, polygons (Fig. $2 \mathrm{~g}$ ) allow charge separation to the corners at low $f$, but at high $f$ this is balanced by attraction across the gap. The conformal shape (Fig. 2f) is better but still too curved at most values of $f$, which can however be corrected with flattened faces and sharpened corners (Fig. 2g), allowing pure dipole distribution of charge.

$4^{4}$

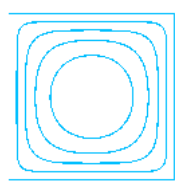

$4.8^{2}$

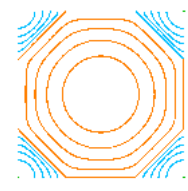

$3^{2} .4 .3 .4$

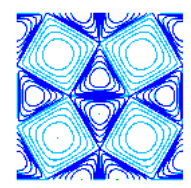

$3^{6}$

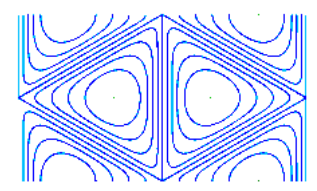

$(3.6)^{2}$

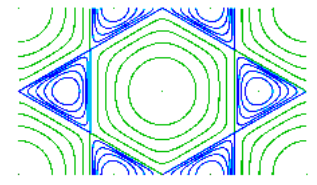

3.4.6.4

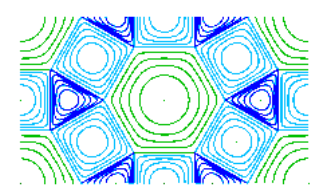

$6^{3}$



$3.12^{2}$

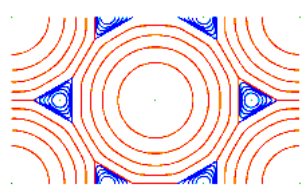

4.6.12

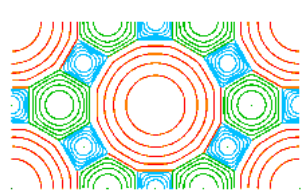

rect(2:1)

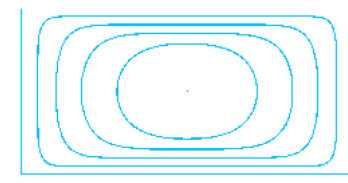

$3^{3} \cdot 4^{2}$

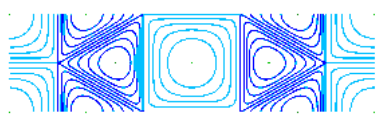

Fig. 3 (color online): Optimized shapes, with contours at intervals of $20 \%$ in $f$. Here the lattices are labelled by their "vertex configuration", where each base numeral is the number of edges of a corresponding face touching the vertex, and the power is shorthand for similar neighbouring vertices. Optimized configurations include the regular tilings $\left(4^{4}\right.$, $\left.3^{6}, 6^{3}\right)$, all of the isotropic semi-regular tilings, and two anisotropic tilings $\left(3^{3} .4^{2}\right.$, and rectangular with $2: 1$ aspect ratio).

Similar trends hold true for several other lattices - Fig. 3 shows optimized shapes (typically to within $10^{-6}$ of MG), including the three regular lattices, and all of the isotropic semi-regular lattices. Even anisotropic lattices can be matched to isotropic MG: as shown I have optimized both rectangles (with 2:1 aspect ratio) and vertex configuration $3^{3} \cdot 4^{2}$. The hexagonal Vigdergauz contours(Vigdergauz 1999) are similar to corresponding contours presented here, but it is notable that the contours for triangular lattices in Fig 3 appear somewhat flatter.

Detailed measurements on Fig 3 confirm that optimal shapes have sharper corners and flatter faces than SC contours. The dominant changes in curvature are related to vertex angle of the particle and its neighbours: the change is largest for sharper shapes (e.g. triangles), and is decreased by rounder neighbours. These changes are summarized in Fig. 4, which provides a succinct parameterization of nearly optimal contours in terms of the 
effective conformal radii (with scaling to the fill factor) - larger radius implies sharper corners \& flatter faces. These contours are close enough to MG for practical purposes ( $\Delta C<10^{-3}$ and often much better).
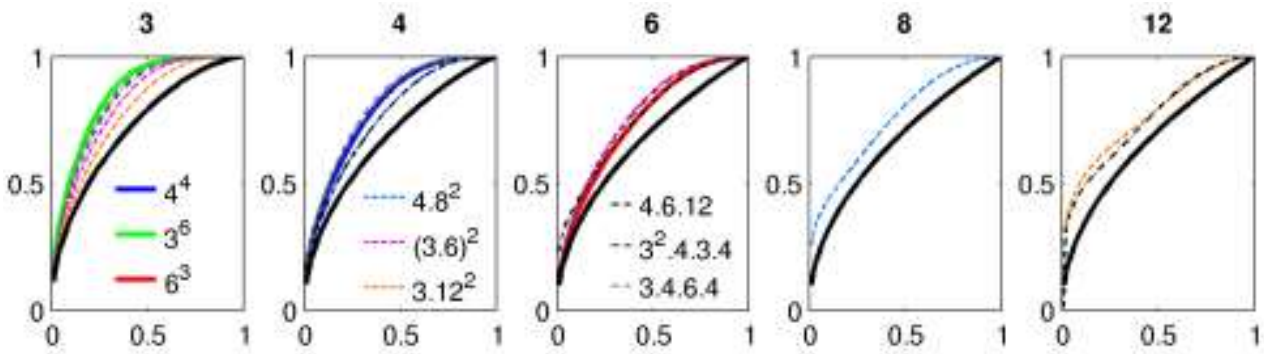

Fig. 4 (color online): Parameterization of nearly optimal contours in terms of the effective conformal radius of individual particles within an array, as a function of fill-factor. Results are grouped by number of sides $(3,4,6,8,12)$ of particles.

The usual conformal radius is shown as a solid black line (below all other curves).

The contours implied by Figure 4 are close enough to MG for practical purposes, as demonstrated by low deviation in Figure 5b. It is also interesting that configurations with good space-filling properties generally have the closest response to MG: in Fig 5a, hexagons are much better than triangles.

(a)

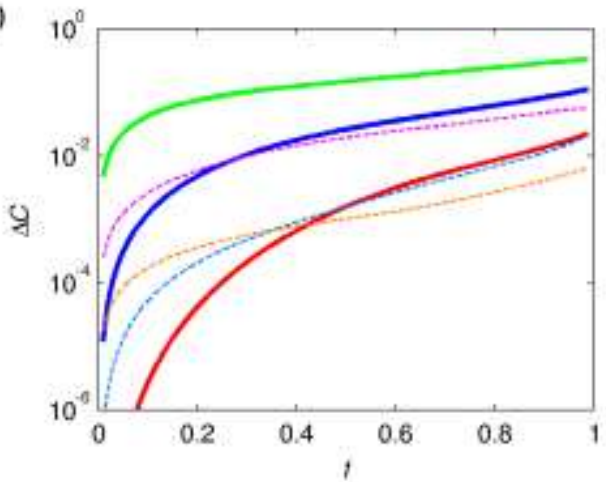

(b)

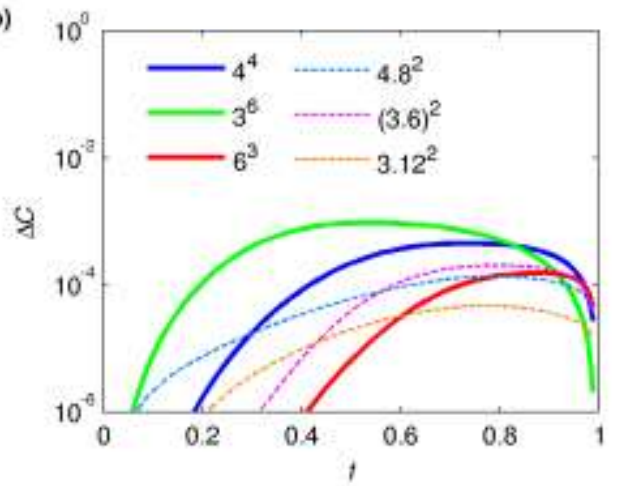

Figure 5 (color online): Comparison of MG deviation as a function of fill factor $f$ for conformal (a), and sharpened conformal (b) contours. The contours in (b) correspond to the parameterization in Figure 4. Circles (not shown) have large deviation and cannot reach $f=1$. Fully optimized results (as in Figure 3) are not shown - typically these have $\Delta C$ $<10^{-6}$. In most of the following results $4^{4}$ from (b) are used.

\section{Electrodynamic deviations}

Finally, I explore electrodynamic deviations from MG on square lattices (circles deviate more in $3^{6}$ and less in $6^{3}$ ) with high index inclusions (networks deviate less). First the deviation compared to MG for dielectric inclusions ( $\varepsilon=12$, approximately that of $\mathrm{Si}$ ) can be examined (Figs $6 \& 7$ ).
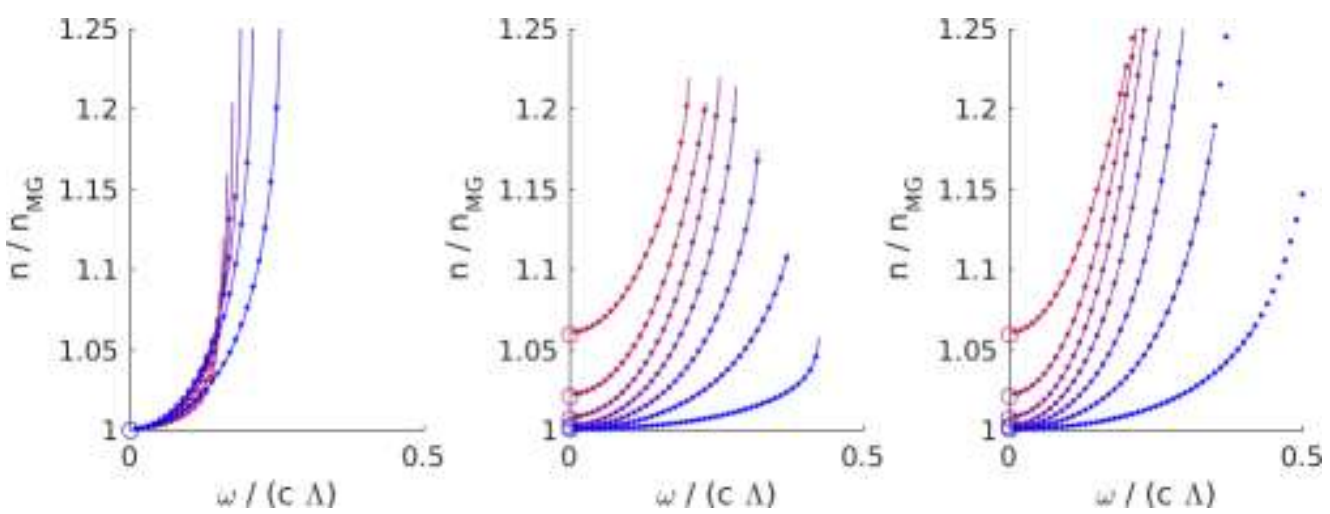

Fig. 6 (color online): Electrodynamic deviation of modes of square arrays of cylinders with $\varepsilon=12$ as a function of period:wavelength at fill factors in steps of 0.1 from $f=0.1$ (blue) to 0.7 (red) near percolation. The polarizations are (from left to right) longitudinal $\mathrm{E}$ (transverse k), transverse $\mathrm{E}$ transverse $\mathrm{k}$, and transverse $\mathrm{E}$ longitudinal $\mathrm{k}$ as in Fig 1. Lines are derived from the first MPB mode up to the edge of the Brillioun zone (actually infinite for longitudinal k), and the dots are COMSOL mode calculations, circles are the BEM static result - all are in good agreement. 

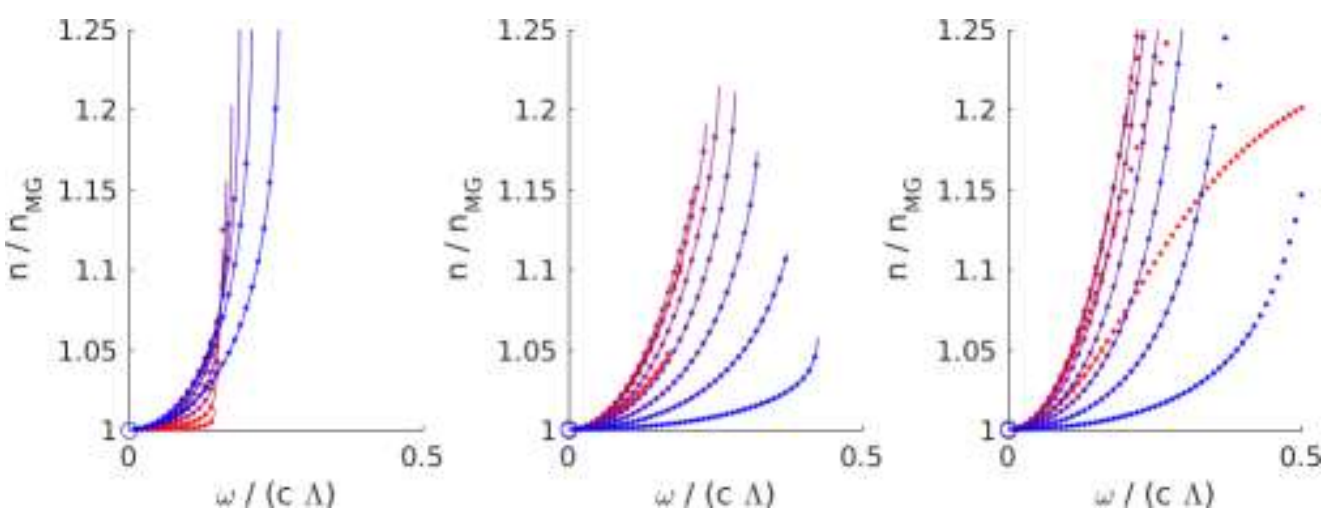

Fig. 7 (color online): Electrodynamic deviation of modes of square arrays of optimized pillars, at $\varepsilon=12$, with all other parameters as in the previous except that the maximum $f=0.9$ (red).

The deviation of circles from MG even at low frequency is evident at high fill-factor in Fig 6, but not for longitudinal $\mathrm{E}$ because $L=0$. As expected there is minimal low-frequency deviation for optimized shapes in Fig 7. All cases show some degree of deviation at higher frequency: this is typically worse for longitudinal $\mathrm{E}$ because the effective index is higher. In general, higher fill-factors also suffer greater deviation due to higher effective index, although $f=0.9$ shows some improvement for longitudinal $\mathrm{k}$ and longitudinal $\mathrm{E}$ is significantly flattened at moderate frequency (Fig 7). The quasistatic multimode dispersion in Fig 6 at $f=0.7$ is about $6 \%$ which is similar in magnitude to the spatial dispersion at a period:wavelength ratio of about 0.1 in Fig 7.

Next we explore issues pertinent to spatial dispersion around plasmon resonances assuming Drude inclusions. In this case, multiple waveguide modes can be excited at a single frequency, but their importance depends on the source distribution, and hence it is useful to consider the response of a realistic experiment such as plane-waves normally incident on finite slabs of pillar media (as represented in Figure 1). Inversion of the complex reflectance and transmittance retrieves effective refractive index and impedance, but these can vary with thickness.

In Figure 8, the results of various methods are compared for three different periods. At small periods, all methods produce similar results as expected: there is a minor difference between eigenmode and retrieved results due to the finite thickness of the latter. As period increases deviations are observed around the resonance, especially between refractive impedance and inverse impedance. Additional prominent deviations in impedance are correlated with low reflection as noted in the literature(Chen, Grzegorczyk et al. 2004). The stronger thickness dependence of impedance suggests that it is more of a surface property of these materials: surface termination effects have also been shown to be important in other metamaterials(Lapine et al. 2012). Again there is minor disagreement between eigenmode and retrieved $n$ due to finite thickness, but the field homogenization at the center of the slab closely matches the eigenmode result. At large periods more than a single mode is excited so the quasistatic approximation is clearly invalidated, and retrieval becomes problematic due to weak transmission. Together these results support the validity of the quasistatic approximation at sufficiently small periods and the premise that the dominant eigenmode is the more reliable measure of bulk properties.
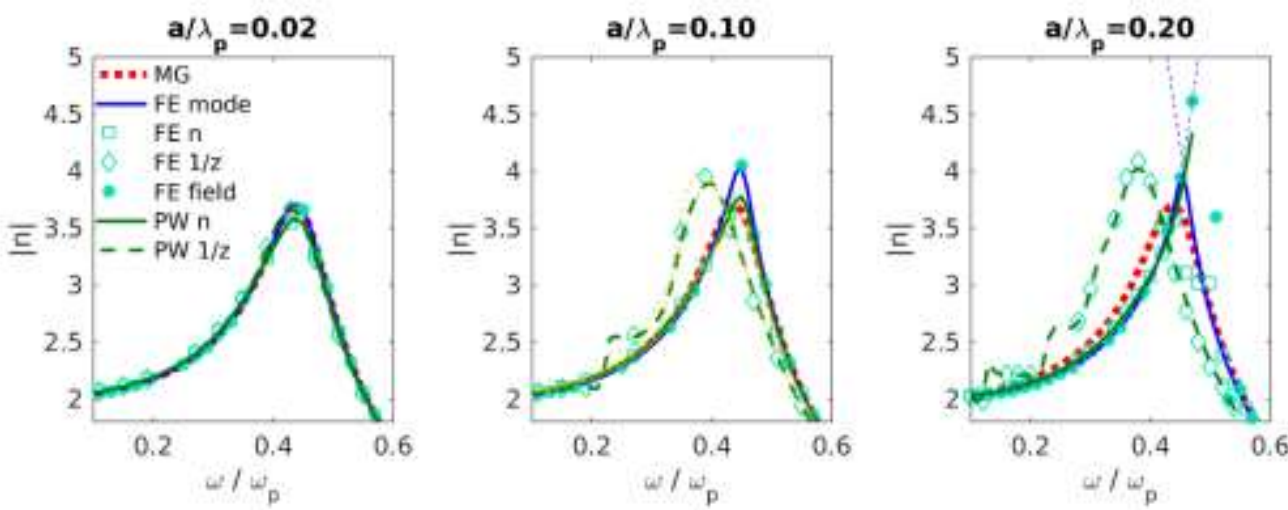

Figure 8 (color online): Comparison of the effective index calculated by various methods: spectra for optimized pillars with $f=0.6$ Drude inclusions $\left(\gamma / \omega_{p}=0.1\right)$ and are shown for three increasing periods. Methods include COMSOL finite element (FE) eigenmode solver, retrieval (n \& 1/z) and central field homogenization on FE results for a finite slab 10 periods thick, and retrieval on the plane-wave code S4. The center panel shows additional results for $5 \& 20$ 
It is evident that spatial dispersion, and indeed disagreement between various definitions of material properties, is greatest around the resonance. To quantify how various parameters contribute to the deviation of the refractive index, the dominant mode at the $M G$ resonant frequency $\omega_{r}$ was surveyed over a range of periods ( $\left.0.02<a / \lambda_{p}<0.2\right)$, Drude damping ratios $\left(0.02<\gamma / \omega_{r}<0.2\right)$ and fill factors $(0.3<f<0.8)$. Results outside this range were omitted from Figure 9 due to numerical limitations or obvious violation of quasistatic assumptions (e.g. at large $a$ ). The observed deviation increases with increasing period $|\Delta n| \sim\left(a / \lambda_{p}\right)^{2}$ which also implies $|\Delta n| \sim\left(\omega / \omega_{p}\right)^{2}$. Further, deviation is increased by decreasing damping ratio $|\Delta n| \sim\left(\gamma / \omega_{p}\right)^{-3}$, and since a MG resonance in a Drude material has $\left|n_{r}\right| \sim\left(\gamma / \omega_{p}\right)^{-3}$ it follows that $|\Delta n| \sim\left|n_{r}\right|^{6}$. The effect of fill-factor is more complicated, because increasing it decreases the resonant frequency $\omega_{r} / \omega_{p} \approx[(1-f) / 2]^{1 / 2}$, but this also increases the effective index $\left|n_{r}\right| \sim\left\{\omega_{r} / \omega_{p}\left[2-\left(\omega_{r} / \omega_{p}\right)^{-2}\right]\right\}^{1 / 2}$ which has already been included above. Since the observed deviation is lower at high and low fill-factors, finally we have that $|\Delta n| \sim\left(a / \lambda_{p}\right)^{2}\left(\omega / \omega_{p}\right)^{2}\left|n_{r}\right|^{6}[f(1-f)]^{2}:$ the interdependence $n\left(\omega_{r}(f)\right)$ allows many possible variants, but this seems to be the most convenient form. This model accounts for much of the $|\Delta n|$ seen in Figure 9, but at low deviations numerical errors are relatively significant, and at high deviations the relationship changes due to significant movement in the peak frequency.
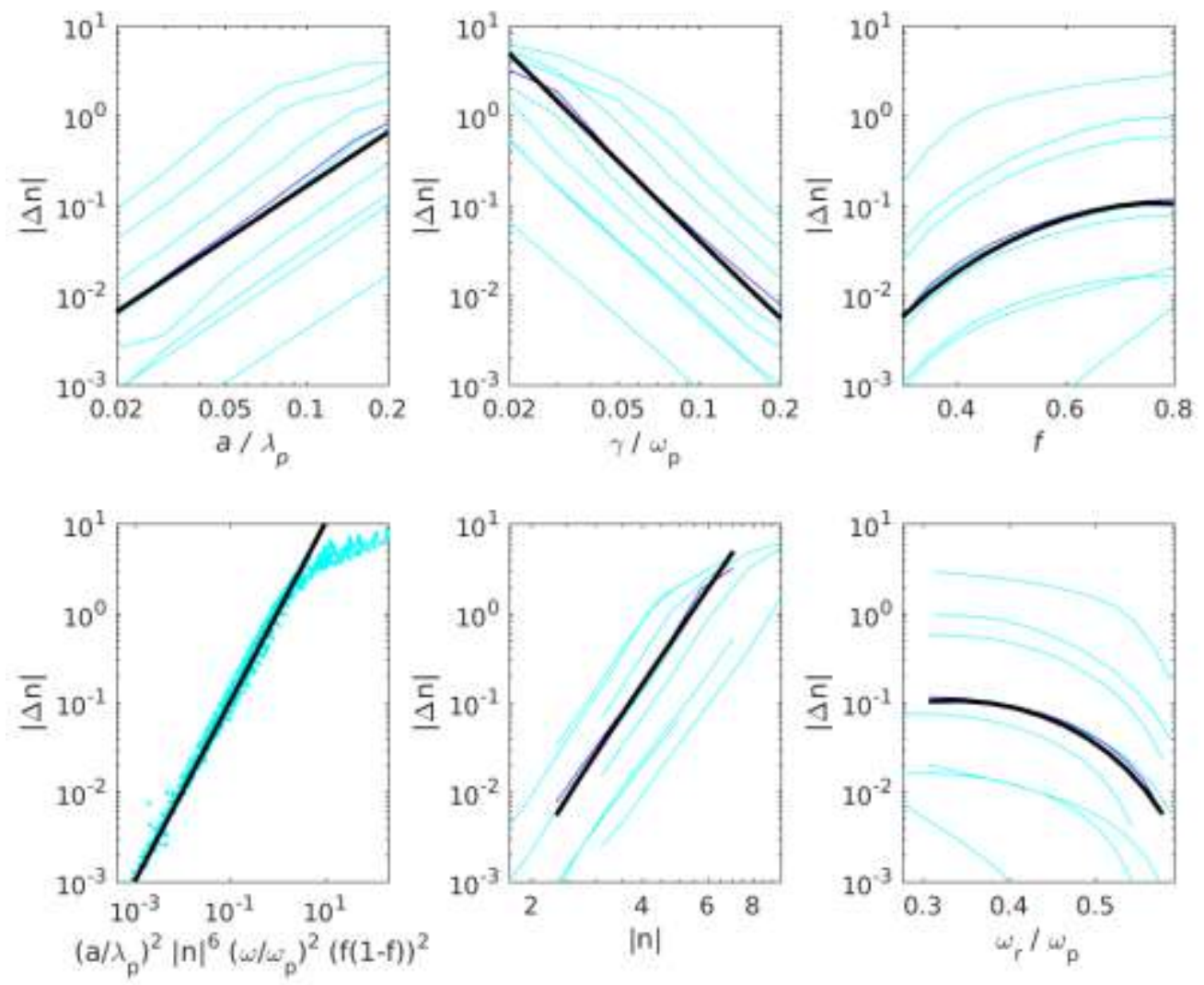

Figure 9 (color online) The electrodynamic deviation $\Delta n$ of the dominant mode from MG at the MG resonance $n\left(\omega_{r}\right)$ as a function of period $a$, Drude damping $\gamma$ and fill-factor $f$, is shown in the top panels. The effect of derived parameters $\omega_{r}(f)$ and $n(f, \gamma)$ is shown in the lower panels. Results for variations in the remaining parameters are shown in light blue, and for reference dark blue lines highlight results intersecting $a=0.05, \gamma=0.1, f=0.5$. The thick black line shows the fit suggested in the text, which is condensed into the lower left panel.

We now consider real materials, which have more complicated permittivity functions. Spatial dispersion in selected materials is demonstrated in Fig 10, at $f=0.7$, where multimode deviations start to become substantial. As expected, electrodynamic deviation increases with period, especially for conducting inclusions (e.g. Ag in Fig 
6c), but in the visible the quasistatic approximation is good at $10 \mathrm{~nm}$ and still better than circles at period $<100$ $\mathrm{nm}$.

(a)

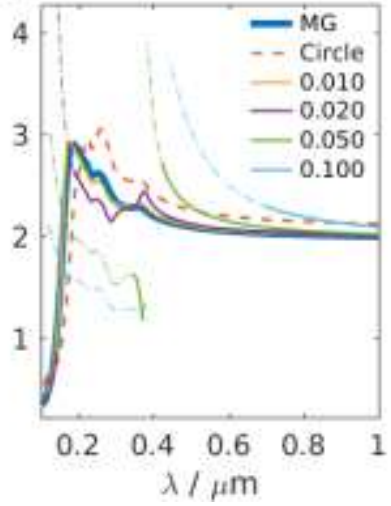

(b)

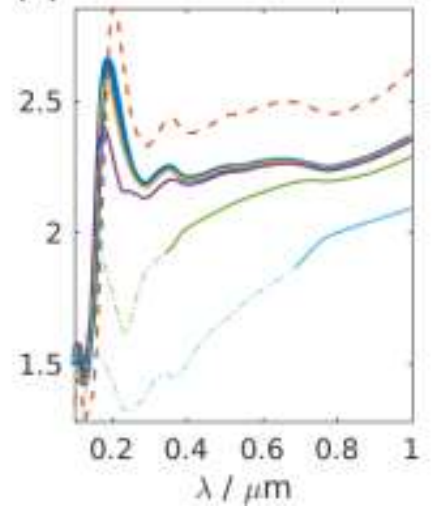

(c)

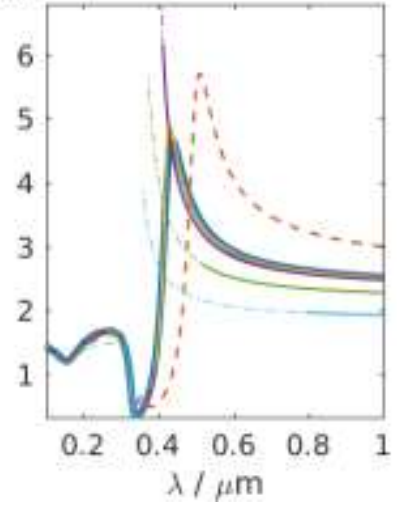

Fig. 10 (color online): Comparison of real refractive index $n$ in square lattices at $f=0.7$ for MG, small circles, and $4^{4}$ shape from Fig 3 at various periods (10 to $100 \mathrm{~nm}$ ). Representative materials include (a) dielectric Si, (b) lossy W, (c) plasmonic Ag, taken from CRC Handbook.

Figure 11 summarizes the effect of fill factor on the resonant deviation. With the exception of $\mathrm{K}$, the results are somewhat different from Fig 9 due to interband transitions. As expected, lossier metals such as W \& Ta have much lower deviation. Unusually, $\mathrm{Al}$ has a prominent interband at low frequency so it is flattened at high $f$. Many metals have interbands at high frequency, so deviation is decreased by increased losses at low fill factor.

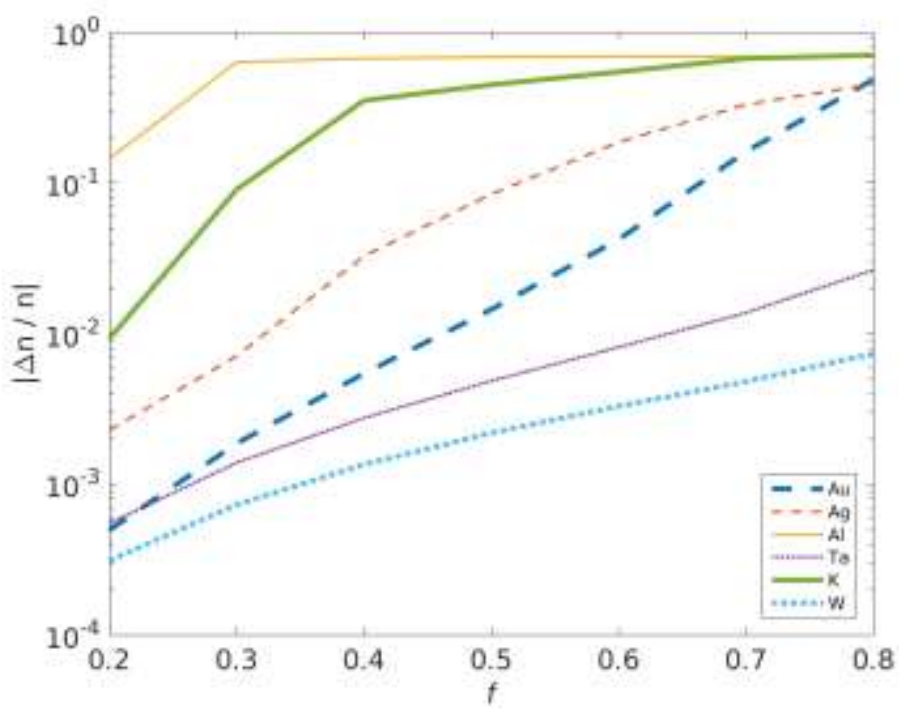

Fig. 11 (color online) Relative resonant deviation from MG as a function of fill-factor $f$, for selected metals with period $a=20 \mathrm{~nm}$. Permittivity from the CRC Handbook.

\section{Conclusion}

There are several special geometries that exactly match quasistatic MG over a much wider range of fill-factors than usually expected. Optimal surface contours are sharper than conformal, with the surface charge spread evenly to match the projection of the normal: this insight should also be applicable to a broad range of other geometries. Electrodynamic effects in these materials place some restrictions on the applicability of a static approach (especially at high permittivities), but the proposed morphologies still offer significant improvement over circular shapes.

\section{Acknowledgments}

This work was supported by ARC DP140102003, NCI usage funded by LE140100002, and conversations with Angelo Garruzzo, Michael Cortie, Geoff Smith, Chris Poulton \& Mikhail Lapine.

\section{References}


Abramowitz M and Stegun I A 1964 Handbook of Mathematical Functions with Formulas, Graphs, and Mathematical Tables New York Dover

Alù A 2011 First-principles homogenization theory for periodic metamaterials Physical Review B 84075153

Belov P, Marques R, Maslovski S, Nefedov I, Silveirinha M, Simovski C and Tretyakov S 2003 Strong spatial dispersion in wire media in the very large wavelength limit Physical Review B 67113103

Bendsøe M P and Sigmund O 1999 Material interpolation schemes in topology optimization Archive of Applied Mechanics 69 635-654

Bergman D J 1979 Dielectric-Constant of a 2-Component Granular Composite - Practical Scheme for Calculating the Pole Spectrum Physical Review B 19 2359-2368

Blaber M G, Arnold M D and Ford M J 2009 Search for the Ideal Plasmonic Nanoshell: The Effects of Surface Scattering and Alternatives to Gold and Silver Journal of Physical Chemistry C 113 3041-3045

Cadman J E, Zhou S, Chen Y and Li Q 2013 On design of multi-functional microstructural materials Journal of Materials Science 48 51-66

Chen X, Grzegorczyk T M, Wu B-I, Pacheco Jr J and Kong J A 2004 Robust method to retrieve the constitutive effective parameters of metamaterials Physical Review E $\mathbf{7 0} 016608$

Davanço M, Urzhumov Y and Shvets G 2007 The complex Bloch bands of a 2D plasmonic crystal displaying isotropic negative refraction Optics Express 15 9681-9691

de Kruijf N, Zhou S, Li Q and Mai Y-W 2007 Topological design of structures and composite materials with multiobjectives International Journal of Solids and Structures $\mathbf{4 4}$ 7092-7109

De Zuani S, Rommel M, Gompf B, Berrier A, Weis J and Dressel M 2016 Suppressed Percolation in Nearly Closed Gold Films ACS Photonics 3 1109-1115

Driscoll T A 1996 Algorithm 756: A MATLAB toolbox for Schwarz-Christoffel mapping ACM Transactions on Mathematical Software 22 168-186

Elser J, Wangberg R, Podolskiy V A and Narimanov E E 2006 Nanowire metamaterials with extreme optical anisotropy Applied Physics Letters 89261102

Fang A, Koschny T and Soukoulis C M 2009 Optical anisotropic metamaterials: Negative refraction and focusing Physical Review B 79245127

Feng W and Whites K W 2001 Quasi-static effective permittivity of periodic composites containing complex shaped dielectric particles Antennas and Propagation, IEEE 49 1174-1182

Fietz C, Urzhumov Y and Shvets G 2011 Complex k band diagrams of 3D metamaterial/photonic crystals Optics Express 19 19027-19041

Fuchs R and Liu S H 1976 Sum rule for the polarizability of small particles Physical Review B 14 5521-5522

Garnett J C M 1904 Colours in Metal Glasses and in Metallic Films Philosophical Transactions of the Royal Society of London A $203385-420$

Golovan' L A, Kashkarov P K and Timoshenko V Y 2007 Form birefringence in porous semiconductors and dielectrics: A review Crystallography Reports 52 672-685

Hetherington J H and Thorpe M F 1992 The Conductivity of a Sheet Containing Inclusions with Sharp Corners Proceedings of the Royal Society of London Series A 438 591-604

Hodgkinson I J, De Silva L and Arnold M D 2005 Inorganic polarizing materials grown by physical vapor deposition Proc SPIE 5870

Huang X, Xie Y M, Jia B, Li Q and Zhou S W 2012 Evolutionary topology optimization of periodic composites for extremal magnetic permeability and electrical permittivity Structural and Multidisciplinary Optimization 46 385-398

Hyun S and Torquato S 2000 Effective elastic and transport properties of regular honeycombs for all densities Journal of Materials Research 15 1985-1993

Inglesfield J E, Pitarke J M and Kemp R 2004 Plasmon bands in metallic nanostructures Physical Review B 69233103

Johnson S G and Joannopoulos J D 2001 Block-iterative frequency-domain methods for Maxwell's equations in a planewave basis Optics Express 8 173-190

Kanungo J and Schilling J 2010 Experimental determination of the principal dielectric functions in silver nanowire metamaterials Applied Physics Letters 97021903

Lapine M, Jelinek L and Marques R 2012 Surface mesoscopic effects in finite metamaterials Optics Express 20 18297-18302 
Liu V and Fan S 2012 S4 : A free electromagnetic solver for layered periodic structures Computer Physics Communications $1832233-2244$

Markel V A and Schotland J C 2012 Homogenization of Maxwell's equations in periodic composites: boundary effects and dispersion relations Physical Review E 85066603

Mayergoyz I D, Fredkin D R and Zhang Z Y 2005 Electrostatic (plasmon) resonances in nanoparticles Physical Review B 72

Mcphedran R C and Mckenzie D R 1980 Electrostatic and Optical Resonances of Arrays of Cylinders Applied Physics 23223 235

McPhedran R C, Poulton C G, Nicorovici N A and Movchan A B 1996 Low Frequency Corrections to the Static Effective Dielectric Constant of a Two-Dimensional Composite Material Proceedings of the Royal Society A 452 2231-2245

Miller O D, Hsu C W, Reid M T, Qiu W, DeLacy B G, Joannopoulos J D, Soljacic M and Johnson S G 2014 Fundamental limits to extinction by metallic nanoparticles Physical Review Letters 112123903

Milton G W 2002 The Theory of Composites Cambridge University Press

Milton G W, McPhedran R C and McKenzie D R 1981 Transport properties of arrays of intersecting cylinders Applied Physics 25 23-30

Pendry J B, Schurig D and Smith D R 2006 Controlling electromagnetic fields Science 312 1780-1782

Perrins W T, Mckenzie D R and Mcphedran R C 1979 Transport-Properties of Regular Arrays of Cylinders Proceedings of the Royal Society of London Series A $\mathbf{3 6 9} 207-225$

Pitarke J M, Inglesfield J E and Giannakis N 2007 Surface-plasmon polaritons in a lattice of metal cylinders Physical Review B 75165415

Poxson D J, Schubert M F, Mont F W, Schubert E F and Kim J K 2009 Broadband omnidirectional antireflection coatings optimized by genetic algorithm Optics Letters $\mathbf{3 4}$ 728-730

Rinnerbauer V, Shen Y, Joannopoulos J D, Soljacic M, Schaffler F and Celanovic I 2014 Superlattice photonic crystal as broadband solar absorber for high temperature operation Optics Express 22 Suppl 7 A1895-1906

Saito M and Miyagi M 1989 Anisotropic optical loss and birefringence of anodized alumina film Journal of the Optical Society of America A 6 1895-1900

Schmidt K and Kappeler R 2010 Efficient computation of photonic crystal waveguide modes with dispersive material Optics Express 18 7307-7322

Sen A K and Torquato S 1989 Effective Conductivity of Anisotropic 2-Phase Composite Media Physical Review B $394504-$ 4515

Shinji N, Mitsunori S, Li-Feng H, Mitsunobu M and Kenji W 1992 Infrared Optical Constants of Anodic Alumina Films with Micropore Arrays Japanese Journal of Applied Physics 313589

Shvets G and Urzhumov Y A 2005 Electric and magnetic properties of sub-wavelength plasmonic crystals Journal of Optics A $7 \mathrm{~S} 23$

Silva A O, Leite I T, Teixeira J M, Araujo J P, Costa J C W A, Giraldi M T R, Jorge P A S and Guerreiro A 2013 Effective medium theory of subwavelength arrays of metallic nanowires: a numerical approach based on modal propagation method Proc SPIE 8785 SPIE

Simovski C R 2011 On electromagnetic characterization and homogenization of nanostructured metamaterials Journal of Optics 13013001

Simovski C R, Belov P A, Atrashchenko A V and Kivshar Y S 2012 Wire metamaterials: physics and applications Advanced Materials 24 4229-4248

Smith D R and Pendry J B 2006 Homogenization of metamaterials by field averaging Journal of the Optical Society of America B 23 391-403

Torquato S and Hyun S 2001 Effective-medium approximation for composite media: Realizable single-scale dispersions Journal of Applied Physics 89 1725-1729

Torquato S, Hyun S and Donev A 2002 Multifunctional composites: optimizing microstructures for simultaneous transport of heat and electricity Physical Review Letters 89266601

Vigdergauz S 1994 Two-dimensional grained composites of extreme rigidity Journal of Applied Mechanics 61 390-394

Vigdergauz S 1996 Rhombic lattice of equi-stress inclusions in an elastic plate The Quarterly Journal of Mechanics and Applied Mathematics 49 565-580

Vigdergauz S 1999 Energy-minimizing inclusions in a planar elastic structure with macroisotropy Structural Optimization 17 $104-112$ 
Vynck K, Felbacq D, Centeno E, Căbuz A I, Cassagne D and Guizal B 2009 All-Dielectric Rod-Type Metamaterials at Optical Frequencies Physical Review Letters 102133901

Wells B M, Zayats A V and Podolskiy V A 2014 Nonlocal optics of plasmonic nanowire metamaterials Physical Review B 89 035111

Whites K W and Feng W 2002 Effects of particle shape on the effective permittivity of composite materials with measurements for lattices of cubes Microwave Theory and Techniques, IEEE 50 1723-1729

Zhou S, Li W and Li Q 2010 Design of 3-D Periodic Metamaterials for Electromagnetic Properties Microwave Theory and Techniques, IEEE $\mathbf{5 8}$ 910-916 